\title{
Gesundheitsverhalten bei Männern - kaum eine Besserung in Sicht
}

\author{
Healthy Lifestyles and Help-Seeking in Males - No Improvement in Sight
}

Bibliografie

DOI $10.1055 / \mathrm{s}-2007-970906$

Psychiat Prax 2007; 34:

367-369 @ Georg Thieme

Verlag KG Stuttgart · New York .

ISSN 0303-4259

Korrespondenzadressen

Prof. Dr. Dr. Wolfgang Rutz

Unit for Public Mental Health, University Hospital, Uppsala,

Fakultät für Soziale Arbeit und Gesundheit, Hochschule Coburg

Ulleråkersvägen 21

75643 Uppsala, Sweden

wolfgang.rutz@akademiska.se

Prof. Dr. Theodor Klotz, MPH

Klinik für Urologie, Andrologie und Kinderurologie

Söllnerstraße 16

92637 Weiden

klotz@klinikum-weiden.de

\section{Pro}

In den osteuropäischen Gesellschaften, die derzeit einen drastischen Übergang erleben, ist ein typisches Krankheits- und Mortalitätsmuster des Mannes zu beobachten. In einigen dieser Länder entstand ein neues Entvölkerungsphänomen, indem sich während der 90er-Jahre die männliche Lebenserwartung teilweise um mehr als 10 Jahre verringerte, während die weibliche Morbidität und Mortalität ziemlich unverändert blieb. Dazu trugen am stärksten ein erhöhtes Vorkommen von Risikobereitschaft, stressbedingten Gefäßkrankheiten und von Suiziden bei.

Wenn man die männlichen Krankheitsraten osteuropäischer Übergangsländer als Ausdruck seelischer Krankheit betrachtet und sie mit der weiblichen Lebenserwartung vergleicht, dann scheinen Frauen in Zeiten der Veränderung geschützter zu sein. Die stressbedingte Morbidität und Mortalität des Mannes spiegelt nahezu seismografisch die Drucklast einer Gesellschaft wider und steht häufig mit der hinterfragten traditionellen Rollenverteilung, einem gesellschaftlichem Statusverlust oder dem Verlust der Rolle als Brotverdiener in Verbindung. Die Mortalität des Mannes korreliert oft stark mit Gewalttätigkeit, Risikoverhalten, Unfällen und stressbedingten Zuständen, sowohl mit kardialen als auch zerebralen Durchblutungsstörungen. Sie ist im Vergleich zur Mortalität der Frauen 5-9-mal erhöht, während letzteres im Schnitt nur 1,5-fach erhöht ist.

Nach Partnerverlust ist die Mortalität schweizerischer Witwer sowie frisch geschiedener Dänen 6-fach höher als die der Frauen. In Europa liegt die männliche Lebenserwartung 5-10 Jahre niedriger als die der Frau, und der zunehmende Abstand der Lebenserwartungen scheint ein zuverlässiger Indikator für die wachsende Stressbelastung einer Gesellschaft zu sein. Die Europäische Kommission betrachtet psychisch bedingte Erkrankungen als „Europas unentdeckte Killer“: Ihr Gesundheitsbeauftragter erklärt, dass die Ge- sellschaft in der wir leben, „Mental Ill Health“ erzeuge, wobei die Sterblichkeit durch psychische und stressbedingte Erkrankungen in Europa offenbar überwiegend eine männliche sei. Anscheinend haben also hauptsächlich Männer Schwierigkeiten, mit den Anforderungen der heutigen „modernen“ Gesellschaft fertig zu werden. Sind sie genetisch anachronistisch programmiert? Ein Verlust an sozialer Integration scheint für Frauen der wichtigste Risikofaktor zu sein, während Männer gegenüber einer Beeinträchtigung von gesellschaftlichem Status und sozialer Würde besonders empfindlich sind.

Auch aus Tierversuchen ist bekannt, dass Männchen am empfindlichsten auf gesellschaftliche Degradierung ansprechen, während Weibchen stärker auf sozialen Entzug und auf den Verlust des Familienzusammenhaltes reagieren. Dementsprechend gibt es Anzeichen, dass stressvolle gesellschaftliche und individuelle Statusverluste, die durch den Verlust der Arbeit und der Rolle als Ernährer verursacht sind, Männer schwerer zusetzen. Frauen dagegen haben die protektive Fähigkeit, während Krisen- und Übergangszeiten soziale Netzwerke aufrechtzuerhalten, Verantwortung für die Familie beizubehalten, und gleichzeitig Kontrolle zu bewahren und Fähigkeiten zu entwickeln, neue Lebensinhalte zu schaffen.

Entgegen landläufiger Annahme gibt es klare Hinweise darauf, dass Männer tatsächlich genauso häufig depressiv sind wie Frauen. Dies wird jedoch oft aufgrund von Alkoholismus, einer begleitenden Drogen- oder anderen Abhängigkeit oder aufgrund von Störungen der Impulskontrolle, von aggressivem und gewalttätigem Verhalten nicht erkannt. Häufig führt dies stattdessen zur Diagnose einer Persönlichkeitsstörung, einer Psychopathie oder einer Abhängigkeit, was kontratherapeutische und nichtempathische Schwerpunkte setzt, und gleichzeitig von der zugrunde liegenden Depression des Mannes ablenkt. 
Anscheinend unterscheiden sich depressive Symptome bei Männern von denen, die bei depressiven Frauen festgestellt werden. Herkömmliche Diagnosekriterien basieren häufig auf subjektiv beschriebenen Depressionssymptomen. Oft sind diese im Bezug auf Männer nicht zutreffend oder unzureichend adaptiert, oft als Folge der alexithymischen Unfähigkeit des Mannes, eine Depression zu spüren und über depressive Symptome zu berichten. Der starke Missbrauch psychotroper Substanzen bei Männern, der durch Hilflosigkeit und Depression verursacht wird, ist oft ein untauglicher Versuch der Selbstmedikation. Durch den gleichzeitigen Mangel an spezifischer Hilfeleistung wird die zugrunde liegende Depression wie in einem Teufelskreis verstärkt.

\section{Ist die niedrigere Depressionsrate bei Männern ein Artefakt?}

In der Volksgruppe der Amish in Nordamerika sind sowohl Aggression und Gewalt als auch Drogenabhängigkeit und Alkoholmissbrauch streng stigmatisiert. In ähnlicher Weise ist in der amerikanisch-jüdischen Gemeinschaft sowie unter israelischorthodoxen Juden Alkoholmissbrauch ein Tabu, und daher beträchtlich seltener als in anderen ethnischen und religiösen Gruppierungen. Wahrscheinlich als Folge hiervon ist die Depressionsrate in diesen Bevölkerungsgruppen bei Männern ebenso hoch wie bei Frauen, und die Suizidrate ist in beiden Geschlechtern gleichermaßen niedrig. Im Gegensatz dazu wird in den Ländern der EU, in denen Alkoholmissbrauch häufiger vorkommt und weniger stigmatisiert ist, die Rate von Depressionen bei Frauen 2-3-mal höher eingeschätzt als bei Männern. Alkoholismus, der Depressionen tarnen kann, ist auch bei russischen Männern 9-mal häufiger als bei Frauen. Das Geschlechterverhältnis von Suiziden zwischen Frauen und Männern liegt in bestimmten russischen Bevölkerungsgruppen bei 1:9, und Depressionen unter Männern werden so gut wie nie festgestellt.

Die Wechselwirkung zwischen Depression, Suizid, Substanzmissbrauch, allgemeiner Gewalt und häuslicher Gewalt wird im „WHO Weltgesundheitsbericht 2001 - Psychische Gesundheit“ und im „WHO Weltgesundheitsbericht 2003 - Gewalt“ genauer ausgearbeitet und führt zu der Annahme, dass nicht nur eine $\mathrm{Zu}$ nahme der männlichen Suizidrate, sondern auch die höhere Prävalenz von Aggression, häuslicher Gewalt und Selbstzerstörung bei Männern mit Depressionen des Mannes zusammenhängen könnte. Diese Probleme könnten durch eine bessere Wahrnehmung, Diagnostik und Therapie von Depressionen bei Männern angegangen werden.

\section{Männer suchen ungern Hilfe}

Es kann problematisch sein, die atypische Depression beim Mann zu erkennen und richtig zu behandeln. Es ist jedoch eine noch größere Herausforderung, depressions- und suizidgefährdete Männer dort zu kontaktieren, wo sie sich häufig aufhalten - oft außerhalb jeglicher sozialer oder Gesundheitsversorgung, da sie weder nach Hilfe suchen, noch sich an Regeln halten, sich oft aggressiv verhalten, ihre Depressionen durch Substanzmissbrauch selbst behandeln, spielen, exzessiven Sport betreiben, der Depression aber auch mit Arbeitssucht oder exzessiver Sexualität zu entkommen suchen.

Ein zusätzliches Problem ist in diesem Zusammenhang, dass die ambulante psychiatrisch-psychotherapeutische Versorgung verständlicherweise den Ansprüchen derjenigen Menschen angepasst ist, die die Dienstleistungen am häufigsten in Anspruch nehmen - also hauptsächlich den Ansprüchen von Frauen. Außerdem sind die Konzepte psychosozialer und psychiatrischer
Hilfe in der Regel von den Anbietern geprägt, welche ebenfalls häufiger weiblich sind. Diese sind sich den spezifischen Problemen der depressiven Psychopathologie des Mannes weniger bewusst und reagieren weniger empathisch darauf.

In vielen Ländern setzt ihre Inanspruchnahme Eigenschaften voraus, die für das weibliche Hilfesuchverhalten typisch sind: Motivation, Einverständnis, Einsicht und die Bereitwilligkeit, sich zu verändern. Dies sind Bedingungen dafür, dass Hilfe angeboten wird, unter gleichzeitigem Ausschluss von Aggressivität und Missbrauch - also Anforderungen, die von gefährdeten Männern oft nicht erfüllbar sind. In vielen Ländern ist die geschlechtsspezifische Ungleichheit institutionalisiert, indem die große Mehrheit der Einrichtungen des Strafvollzugs und der gerichtlichen Psychiatrie von Männern in Anspruch genommen wird, während $80 \%$ des stützenden ambulanten psychiatrischen und psychischen Gesundheitswesens von Frauen genutzt wird.

Es sollte daher ein eindeutiges Interesse dafür entwickelt werden, dass sich momentan in vielen Gesellschaften dieser Welt eine umgreifende Veränderung der Geschlechterrollen vollzieht - ein klares Interesse für die besonders starke Ausgesetztheit des Mannes gegenüber Stressfaktoren und gesellschaftlicher Veränderungen.

Referenzen: Beim Autor.

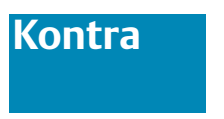

Die Ausgangslage in Hinblick auf das Gesundheitsverhalten von Männern war vor ca. 10 Jahren zweifellos deprimierend: Die geschlechtsspezifische Lebenserwartung ist für Männer um ca. 6,5 Jahre kürzer als für Frauen. Vor allem in den Bereichen der kardiovaskulären Mortalität, der Onkologie des Erwachsenenalters und der verhaltensspezifischen Mortalität (Unfälle, Suizide etc.) stellen Männer das benachteiligte Geschlecht dar. Ein geschlechtsspezifischer Lebenswartungsunterschied zuungunsten der Männer lässt sich für fast alle Industrienationen nachweisen. Erfreulich ist, dass in den letzten 5 Jahren bei weiter deutlich steigender Gesamtlebenserwartung beider Geschlechter sich die Schere zwischen männlicher und weiblicher Lebenserwartung wieder tendenziell verkleinert. Es tut sich also etwas. Wir wissen allerdings noch nicht, ob dieser Trend auf einer besseren geschlechtsspezifischen Versorgung bzw. Sozialisation basiert oder Folge besseren männlichen Gesundheitsverhaltens ist.

\section{Akzeptanz geschlechtsspezifischer Betrachtungen}

In den letzten Jahren hat sich in Bezug auf Männergesundheit viel getan. Wir wissen jetzt, dass die geschlechtsspezifischen Mortalitäten stark mit dem Alter variieren. Dies gilt insbesondere für die Altersgruppen der unter 50-Jährigen. So sind Männer für verhaltensbezogene Mortalitäten vor allem in der Altersklasse der jungen Erwachsenen vulnerabel. Im reifen Erwachsenenalter nach dem 50. Lebensjahr dominieren Herz-Kreislauf-Erkrankungen für Mortalitätsbetrachtungen. Ab dem 75. Lebensjahr gleichen sich die Mortalitätsziffern von Männern und Frauen für die meisten Erkrankungen an. Es existieren eine Reihe von Hinweisen, dass die Basis des geschlechtsspezifischen Lebenserwartungsunterschiedes in der Jugend, d. h. vor dem 20. Lebensjahr gelegt wird. Dies unterstreicht die Hypothese eines genetisch assoziierten Einflusses auf die Lebenserwartung bzw. die Mortalität, der durch verhaltens- und umweltbezogene Einflüsse modifiziert wird. Dies wiederum bedeutet, dass Familienpolitik und genau genommen die meist über Mütter vermittelte Gesundheitserziehung entscheidenden Einfluss auf das spätere Le- 
ben eines Mannes hat. Während die Gesundheitserziehung von Jungen noch verbesserungswürdig ist, hat sich bei Männern in den Altersgruppen der 30-60-Jährigen durchaus eine Verbesserung ergeben. Dies zeigen u.a. die zunehmende Akzeptanz von Vorsorge und Fitnessangeboten.

\section{Männlicher Lebensstil und soziales Immunsystem}

Es ist gut belegt, dass männliche Verhaltensweisen häufig riskant und gesundheitsschädigend sind. Ernährungs- und Suchtverhalten sind nur zwei Beispiele. So sind Männer hinsichtlich Body-Mass-Index (BMI) als Bezugsgröße in allen Altersklassen adipöser. „Frauen reden über Diäten - Männer sind dick.“ Allerdings greift die alleinige verhaltensbezogene Argumentation für die höhere Mortalität von 50-70-jährigen Männern zu kurz, auch wenn falsches Ess- und Bewegungsverhalten wichtige Faktoren sind. So kann der verhaltensbezogene Ansatz nicht die höhere männliche Sterblichkeit bei männlichen Kleinkindern und die späte Angleichung der geschlechtsspezifischen Sterblichkeit bei den Hochbetagten erklären. Es stellt sich die Frage, in welchem Ausmaß diese genetischen Assoziationen für die geschlechtsspezifische Mortalität verantwortlich sind. Ein auffälliger geschlechtsspezifischer Unterschied ist die hormonelle Situation, die letztlich genetisch bedingt ist. Einige Untersuchungen machen einen Einfluss der geschlechtsspezifischen Hormonsituation für Risikoverhalten und bestimmte krankheitsspezifische Mortalitäten wahrscheinlich. Ein gutes Beispiel stellt der Straßenverkehr dar. Zwar sind vor allem ,junge Männer“ die risikobereiteren Autofahrer, aber auch hier hat sich in den Industrienationen in den letzten Jahren eine Verbesserung ergeben. Die Unfallzahlen im Straßenverkehr mit Todesfolge gehen bedingt durch Aufklärungskampagnen und intelligentere Ansprache der Zielgruppen mit dem Ziel der Risikoprävention tendenziell zurück.

Soziale Netzwerke haben die Funktion individuelle Belastungen der Umwelt abzufedern. Die sozialen Netzwerke von Männern sind in der Regel berufsorientiert. Daher besteht eine höhere Anfälligkeit des sozialen Netzwerkes bei Arbeitsplatzverlust oder Erkrankung. So muss die Bedeutung von sozialen Netzwerken bei älteren Männern für die Morbidität und Mortalität hoch angesetzt werden. Hinweisend hierfür ist die ansteigende Morbidität nach Arbeitsplatzverlust, aber auch nach Beginn des Ruhestandes. Typisch männlich ist die Fixierung auf eine einzelne Bezugsperson - in der Regel die Ehefrau. In die gleiche Richtung deuten Untersuchungen, die zeigen, dass die Ehe einen gesundheitsprotektiven Wert für Männer aufweist. Daraus resultiert die These, dass berufsunabhängige soziale Aktivitäten, die in der Regel freizeit-, hobby-, vereins- oder sportorientiert sind, besonders für Männer gesundheitsprotektiv wirken. Hier verbessert sich die Situation bei den 30-60-jährigen Männern - allerdings mit einem deutlichen sozialen Schichtgefälle.

\section{Geschlechtsspezifische Lebensqualität und Umgang mit dem eigenen Körper}

Eine Schwäche der Betrachtungen zur geschlechtsspezifischen Mortalität liegt in der Betonung der Lebenserwartung. Diese Gewichtung ist kritisch zu hinterfragen. So muss eine höhere Lebenserwartung bei Frauen keinesfalls mit einer höheren Lebensqualität einhergehen. Es ist zudem wahrscheinlich, dass Lebensqualität ausgeprägten geschlechtsspezifischen Einflüssen unterworfen ist, die je nach Lebensalter oder -phase variieren. Einige Untersuchungen deuten darauf hin, dass aktivitätsorientierte männliche Rollenmuster zu einer höheren Zufriedenheit und subjektiv höheren Lebensqualität führen. Der Preis für die höhere Lebensqualität der Männer wäre dann - salopp formuliert eine geringere Lebenserwartung. Unterstützt wird diese These dadurch, dass bei vielen Männern die bewusste Einstellung „Lieber kurz und gut als länger und schlechter" nachweisbar ist. So sind einige Risikoverhaltensweisen teilweise zu erklären, die einen unmittelbar erlebten Lebensqualitätsgewinn durch Erfolg und Ansehen (z.B. Risikosportarten, Anabolikaabusus) ermöglichen.

Die Gesundheitsvorsorge für Männer darf in Deutschland als noch unterentwickelt mit Besserungstendenz angesehen werden. Die Frauenbewegung hat durchaus Veränderungen bei traditionellen Denkmustern bewirkt. Allerdings hat sie nicht selten versucht, dem Männerbild weibliche Eigenschaften und Denkweisen überzustülpen. Der neue Mann soll einfühlsam, empfindsam und friedliebend sein. Dennoch soll er auch in Gesellschaft, Beruf und Familie seinen „Mann“ stehen, denn sonst verliert er an Attraktivität. In diesem Zusammenhang ist wesentlich, dass es in fast allen bekannten Gesellschaften einen Zusammenhang zwischen männlichem Habitus und attraktiver Männlichkeit gibt.

Mit dieser Ausrichtung auf sozialen Status und Erfolg hängt zusammen, dass Männer dazu neigen, ihren Körper als Werkzeug zum Erreichen eines Ziels zu instrumentalisieren. Der Körper muss funktionieren in Beruf, Sport bzw. Sexualität. Daraus erklärt sich, dass rücksichtloses Verhalten gegenüber dem eigenen Körper bei Männern an der Tagesordnung steht. Doch liegen darin auch Chancen, männliche risikoreiche Verhaltensmuster oder Lebensstile zu ändern. Hier hat eine kindgerechte Familienpolitik eine entscheidende Bedeutung für das spätere Erwachsenenalter. Dies gilt offenbar für das männliche Geschlecht in besonders hohem Maß. Entscheidend ist, gesellschaftliche Wandlungsprozesse zu fördern, die Gesundheit und gesundheitsbewusstes Verhalten bei Männern mit sozialem Status und Karriere positiv verknüpfen. Dann wird sich männliches Verhalten von alleine ohne den erhobenen Zeigefinger ändern. Männlichkeit würde sich dann u. a. in einem höheren Gesundheitsbewusstsein äußern, um z.B. das Ziel „sozialer Aufstieg“ zu erreichen. Erste Ansätze lassen sich zweifellos in der Generation der 30-60-jährigen Männer erkennen - dennoch bleibt noch viel tu tun.

\section{Literatur}

1 Brandes H. Männlicher Habitus und Gesundheit. Blickpunkt Der Mann 2003; $2: 10-13$

2 Enstrom JE, Kanim LE, Klein MA. Vitamin C intake and mortality among a sample of the United States population. Epidemiology 1992; 3: 193 202

3 Klotz T, Hurrelmann K, Eickenberg HU. Der frühe Tod des starken Geschlechts. Dt Ärzteblatt 1998; 95: 460-464

4 Kulik JA, Mahler HIM. Social support and recovery from surgery. Health Psychology 1989; 8: $221-238$

5 Martikainen $P$, Valkonen T. Mortality after the death of a spouse: rates and causes of death in a large finnish cohort. Am J Public Health 1996; 86: $1087-1093$

6 Neubauer G. Wie geht's den Jungs? Jungengesundheit und Aspekte einer jungenbezogenen Gesundheitsförderung. Der Mann 2003; 1: $24-$ 28

7 Rubinow DR, Schmidt PJ. Androgens, Brain and Behaviour. Am J Psychiatry 1996; 153: 974-984

8 Schneider J, Cebrat S, Stauffer D. Why do women live longer than men? A Monte Carlo simulation of Penna-type models with X and Y chromosoms. Int J Mod Physics C 1998; 5: 721 - 725

9 Statistisches Bundesamt Deutschland. Daten des Gesundheitswesens 2002, www.destatis.de/basis/d/gesu 